\title{
OBESIDADE JUVENIL COM ENFOQUE NA PROMOÇÃO DA SAÚDE: revisão integrativa
}

\author{
I zaildo T avares LU NA a, Rosa A parecida N ogueira M ORE IRA ${ }^{b}$, K elanne Lima da SI LVAc, \\ Joselany Áfio CAETAN O d, Patrícia N eyva da Costa PINHEIRO d, Cristiana Brasil de Almeida REBOU ÇAS
}

\section{RESUMO}

Foi realizada uma revisão integrativa com o objetivo de sintetizar as contribuições das pesquisas em Enfermagem sobre obesidade juvenil com enfoque na promoção da saúde. A través do levantamento bibliog ráfico foram identificados 20 artigos considerando os critérios: data, idioma e os descritores Enfermagem, adolescente, obesidade e promoção da saúde. 0 periódico P ediatric N ursing J ournal apresentou 7 (35\%) artigos. As publicações indexadas ocorreram, nos últimos dez anos, nos idiomas português e inglês. Os resultados evidenciaram a construção do conhecimento científico da E nfermagem para o desenvolvimento de estratégias com enfoque na promoção da saúde na obesidade juvenil e, assim, contribuir para o desenvolvimento da profissão. Consideramos que a visualização do risco cumulativo que a obesidade juvenil apresenta em tor nar o sujeito um adul to obeso éum dado precioso para que o planejamento das ações de enfermagem direcionadas a essa população seja implementado e al cance resultados efetivos.

D escritores: A dolescente. O besidade. Promoção da saúde.

\section{RESUMEN}

F ue desarrollada una revisión integradora para sintetizar las contribuciones de las investigaciones en E nfermería sobre obesidad juvenil con foco en la promoción de la salud. A través de levantamiento bibliográfico fueron identificados 20 artículos, considerando los criterios: fecha, idioma y los descriptores E nfer mería, adolescente, obesidad y promoción de la salud. E I periódico P ediatric N ursing J ournal mostró siete artículos (35\%). L as publicaciones indexadas ocur rier on en los últimos diez años, en portugués e inglés. L os resultados evidenciaron la construcción del conocimiento científico de E nfermería para el desar rollo de estrategias con foco en la promoción de la salud en obesidad juvenil y, así, contribuir al desar rollo de la profesión. Consideramos que la visualización del riesgo cumulativo que la obesidad juvenil muestra en hacer del sujeto un adulto obeso es un dato precioso para la implementación de la planificación de acciones de enfermería dirigidas a esa población y el alcance de resultados efectivos.

Descriptores: A dolescente 0 besidad. P romoción de la salud.

T ítulo: 0 besidad juvenil con foco en la promoción de la salud: revisión integradora.

\section{ABST RACT}

This integrative review summarizes nursing researches that contribute to study juvenile obesity with a focus on health promotion. 20 articles were identified in a bibliographical survey that followed the criteria: date, language, and the descriptors: nursing, adolescent, obesity and health promotion. The Pediatric $N$ ursing J ournal published seven of these papers (35\%). Indexed papers w ere published in the last ten years in Portuguese and E nglish. R esults show ed the construction of scientific know ledgein nursing that developed health promotion strategies in cases of juvenile obesity, thus contributing to the development of the profession. Showing the cumulative risk that juvenile obesity presents of turning subjects into obese adults is a precious resource to plan nursing actions for this population, and for these actions to achieve ef fective results.

D escriptors: A dol escent. 0 besity. $H$ ealth promotion.

T itle: J uvenile obesity with a focus on health promotion: integrative review.

\footnotetext{
a Enfermeiro, M estrando do Programa de Pós-G raduação em Enfer magem da U niversidade F ederal do Ceará (U FC), Professor Substituto do Departamento de Enfermagem da UFC, Bolsista Capes, Fortaleza, Ceará, Brasil.

b Enfer meira E specialista em U nidade de Terapia Intensiva, M estranda do Programa de Pós-G raduação em E nfer magem da U FC, Fortaleza, Ceará, Brasil.

'Enfermeira, M estranda do Programa de Pós-G raduação em Enfermagem da U FC, Fortaleza, Ceará, Brasil.

d D outora em Enfermagem, Professora A djunta do D epartamento de Enfermagem da U FC, Fortaleza, Ceará, Brasil.

e D outora em Enfermagem, Pós-D outoranda do Programa de Pós-G raduação em E nfermagem da UFC, Professora Colaboradora do Programa de Pós-G raduação em Enfermagem do D epartamento de Enfermagem da U FC, Bolsista CN Pq, Fortaleza, Ceará, Brasil.
} 


\section{INT RODUÇÃO}

A obesidade é um distúrbio associado a um risco elevado de morbi-mortalidade devido à diversidade de complicações que produz, juntamente com a deterioração da qualidade de vida(1).

Crianças e adolescentes obesos serão, em potencial, adultos obesos. 0 risco cumulativo para a obesidade na idade adulta varia de 2,33 para crianças com peso acima do percentil 90 , nos seis primeiros meses de vida, até 6,55, observado em meninos e meninas entre 10 e 13 anos de idade. Foi observada a prevalência de obesidade em $21,1 \%$ das meninas e 8,9 \% dos meninos entre 10 e 18 anos, em São Paulo(2).

Vários fatores influenciam o comportamento alimentar. Entre eles há os fatores externos, como a unidade familiar e suas características e fatores internos, como necessidades e características psicológicas, imagem corporal, valores e experiências pessoais, autoestima, preferências alimentares, saúde e desenvolvimento psicológico(3).

0 enfermeiro desenvolve ações de prevenção primária no controle da obesidade através de ações educativas. Com o aumento do número de obesos juvenis, os cuidados com essa população vêm se tornando desafiadores em todas as fases da vida, devido à dificuldade em se manter a disciplina alimentar.

A atuação integrada dos membros da equipe de saúde é de grande importância para conquistas futuras. T ambém é evidente que essas medidas só serão al cançadas se houver participação das famílias, das escolas e das comunidades, em um esfor ço conjunto da sociedade e do governo(4).

Os aspectos sociais e emocionais da obesidade são imediatos e têm impacto negativo sobre 0 bem-estar do adolescente, além de consequências à saúde do seu corpo a longo prazo. São previstos baixa satisfação corporal, baixa autoestima, aumento da depressão e ideação suicida ${ }^{(5)}$. Crianças obesas são mais susceptíveis de se tornarem adultos obesos, o que impõe enormes custos para o sistema de saúde. Assim, há uma necessidade urgente de buscar, desenvolver, testar e traduzir as intervenções baseadas em evidências para o tratamento e prevenção da obesidade juvenil(6).

0 conhecimento de estratégias acerca do crescimento numérico da obesidade juvenil é um componente importante para o desenvolvimento de esforços na sua prevenção. M uitas evidências na pre venção da obesidade derivam de um número modesto de publicações, a maioria das quais abrange, em sua abordagem, estratégias individuais e no âmbito escolar. Há pouca orientação na literatura sobre o desenvolvimento de ações concretas na área da saúde com enfoque em enfer magem ${ }^{(7)}$.

Sabemos a importância dos conhecimentos acerca da temática para estruturar a elaboração de estratégias das intervenções de enfermagem nesse público. $T$ al fato justifica o interesse em desenvolver uma revisão integrativa sobre a produção científica neste assunto, para identificar as investigações já produzidas, além de auxiliar no desenvolvimento de futuras pesquisas.

A creditamos que adolescentes obesos necessitam de intervenções imediatas. E ssa situação leva 0 enfermeiro a estimar a necessidade de criação de estratégias de promoção da saúde direcionando o planejamento e as intervenções. N este intuito, este estudo poderá contribuir para a construção de novas pesquisas e para o planejamento de um cuidado de enfermagem junto a essa população. Daí surge a seguinte pergunta norteadora: como as pesquisas de enfermagem têm contribuído para esclarecer a obesidade juvenil e as ações de enfermagem?

A resposta a essa questão podem colaborar para que atuações efetivas de promoção da saúde sejam realizadas. A importância desse achado poderá aprimorar a atuação do enfermeiro. Os objetivos deste estudo foram caracterizar os trabaIhos produzidos e sintetizar sua contribuição para o enfoque da promoção da saúde na obesidade juvenil.

\section{METODOLOGIA}

Trata-se de revisão integrativa da literatura acerca da obesidade juvenil. A revisão integrativa é uma ferramenta importante no processo de comunicação dos resultados de pesquisas, facilitando sua utilização na prática clínica, uma vez que proporciona uma síntese do conhecimento já produzido e fornece subsídios para a melhoria da assistência à saúde. Oferece, ainda, aos profissionais de diversas áreas de atuação na saúde, o acesso rápido aos resultados relevantes de pesquisas que fundamentam as condutas ou a tomada de decisão, proporcionando um saber $\mathrm{crítico}^{(8)}$.

Para a operacionalização desta revisão utilizamos as seguintes etapas: definição dos critérios 
de inclusão e exclusão, definição das informações a serem extraídas dos estudos selecionados (categorização dos estudos), análise e interpretação dos dados, avaliação dos estudos incluídos na revisão integrativa e apresentação da revisão/ síntese do conhecimento.

0 levantamento bibliográfico foi realizado nos meses de abril e maio de 2010, e o período de inclusão dos estudos entre 2000 e 2010, em duas bases de dados indexadas: L iteratura L atino-A mericana em Ciências da Saúde (LILACS) e M edical $L$ iterature Analysis and R etrieval System 0 nline (M EDLINE). Para o levantamento dos artigos utilizaram-se os descritores enfermagem, adolescente e obesidade, dos D escritores em Ciências da Saúde/ M edical Subject H eadings (DECS/ M ESH) na Biblioteca V irtual em Saúde (BVS).

Os critérios utilizados para o levantamento bibliográfico foram artigos completos, disponíveis nas referidas bases de dados, nos idiomas português e inglês. Destacamos que em virtude das características específicas para 0 acesso das bases de dados selecionadas, a localização dos artigos na íntegra constituiu limitações desta revisão, justificando a quantidade reduzida dos estudos selecionados, pois muitas pesquisas disponibilizavam apenas o resumo $0^{(9)}$. Os critérios de exclusão foram artigos repetidos que estivessem em mais de uma base de dados e que não contemplassem os objetivos do estudo.

$\mathrm{Na}$ busca inicial, 45 artigos foram encontrados, sendo 44 na base M ED LINE e 1 na LILACS. Dos artigos, por meio da busca do texto completo, excluíram-se 15 publicações do M EDLINE e um do LILACS por só apresentarem o resumo. Destes, restaram 28 artigos, os quais foram lidos na íntegra. No entanto, apenas 20 artigos responderam à questão norteadora e fizeram parte da presente revisão.

A coleta de informações se deu a partir de um instrumento adaptado ao tema em estudo, o qual foi preenchido para cada artigo da amostra final da revisão ${ }^{(10)}$. 0 instrumento utilizado apresentou as seguintes informações: título do artigo, ano de publicação, nome do periódico, base de dados, objetivos, delineamento, metodologia, características dos estudos, área temática do artigo, resultados e conclusões, bem como os níveis de evidência dos artigos.

A essência da revisão integrativa é a categorização dos estudos e, neste sentido, procedemos à apresentação dos resultados e discussões das informações de forma descritiva e por meio da construção de tabelas que contêm o delineamento do estudo, as características do estudo e as áreas temáticas das pesquisas.

\section{RESULTADOS E DISCUSSÕES}

Os resultados e as discussões foram apresentados pela ordem das questões do formulário da coleta de dados. F izeram parte da pesquisa 20 periódicos descritos a seguir: T he J ournal of $\mathrm{School}$ N ursing, Pediatric N ursing J ournal, J ournal of Advanced N ursing, N ursing $M$ anagement, $P$ ublic $\mathrm{H}$ ealth $\mathrm{N}$ ursing, J ournal of the A merican Academy of $\mathrm{N}$ urse Practitioners, J ournal of M idwifery \& Women's $\mathrm{H}$ ealth, American J ournal of $\mathrm{N}$ ursing, J ournal of Pediatric $\mathrm{H}$ ealth $\mathrm{Care}$, J ournal of $\mathrm{N}$ ursing Scholarship, N ursing F orum, T he ABN F J ournal e British J ournal of $G$ eneral Practice.

Observamos que o periódico onde foi publicado o maior número de artigos com a temática investigada foi o P ediatric N ursing J ournal, com 35\% (7) dos estudos. Acreditamos que essa maior frequência se explique por se tratar de uma população específica e pelo enfoque da enfermagem como área de atuação. E videnciamos publicação em periódicos distintos envolvendo as mais diversas áreas da saúde, comprometendo a rápida atualização desse conhecimento pela enfermagem.

No que se refere ao ano de publicação, quanto à frequência e porcentagem dos estudos sobre enfer magem e obesidade juvenil identificados nos periódicos nacionais e inter nacionais indexados no M EDL INE, no período de 2000 a 2010, visualizamos em $45 \%$ (9) das pesquisas desenvolvidas pela enfermagem a partir do ano 2000. A pesar de a obesidade ser uma epidemia mundial e a população jovem com obesidade aumentar a cada ano, as publicações de enfermagem encontradas no período de 2009 a 2010 foram apenas $25 \%$ (5) dos estudos das 20 pesquisas, mostrando, com isso, que apesar da relevância da temática, ainda temos um número insipiente de publicações da enfermagem sobre o tema.

A análise dos estudos que compuseram essa revisão evidenciou que $15 \%$ (3) dos periódicos apresentam qual is A 1, 2 (10\%) qual is A2, $8(40 \%)$ com qualis B1, 3 (15\%) em B2 e 4 (20\%) classificados em B3 e B4. Com estes dados percebemos que $80 \%$ (16) dos artigos que serviram para a construção 
desta síntese foram publicados em revistas bem pontuadas na classificação das bases bibliográficas inter nacionais, o que demonstra a qualidade dos periódicos analisados neste estudo.

No que se refere ao delineamento do estudo, verificamos que as pesquisas quantitativas foram as mais frequentes, conforme mostra a T abela 1.

T abela 1 - Delineamento dos estudos indexados no MEDLINE no período de 2000 a 2010, utilizando os descritores enfer magem, obesidade e adolescência. Fortaleza, CE , 2010.

\begin{tabular}{lcr}
\hline Delineamento do estudo & $\mathbf{n}$ & $\%$ \\
\hline Quantitativo/ experimental(11,12) & 2 & 10 \\
Quantitativo/ quase experimental(13,14) & 2 & 10 \\
Quantitativo/ não experimental|(5,6,15-18) & 6 & 30 \\
Quantitativo/ revisão bibliográfica(19-27) & 9 & 45 \\
Qualitativo/ outro(28) & 1 & 5 \\
\hline T otal & $\mathbf{2 0}$ & $\mathbf{1 0 0}$ \\
\hline
\end{tabular}

Observamos, na T abela 1, que apenas 5\% (1) dos estudos envolvem o delineamento qual itativo. $\mathrm{N}$ ão foi observado interesse de abordagem da enfermagem nesse tipo de pesquisa, mas destacamos a importância dessa abordagem por gerar conhecimentos, para a enfermagem, de fenômenos que não podem se tornar objetivos, e os fenômenos humanos constituem o foco de interesse da profissão(29).

Chamou atenção o fato desta revisão evidenciar 95\% (19) dos artigos com método quantitativo. Esses estudos levam a descobertas que proporcionam a validação da prática clínica e fundamentos lógicos para mudar aspectos na prática ${ }^{(30)}$. Além disso, as pesquisas com delineamento experimentais e quase experimentais são fundamentais para estabelecer a prática baseada em evidências.

E $m$ síntese, as pesquisas qualitativas e quantitativas se complementam porque geram diferentes tipos de conhecimentos importantes para a prática da enfermagem, o que determina o tipo de delineamento mais adequado para conduzir a natureza do problema. A mbos os delineamentos requerem experiência do observador, rigor no desenvolvimento do estudo, além de gerar conhecimento para o desenvolvimento da profissão(31). D estacamos que $45 \%$ (7) dos estudos são de revisão bibliográfica, 0 que contribui para o aprofundamento do tema estudado, dando subsídio para o desenvolvimento de outros tipos de pesquisa.
Ao anal isar a T abela 2, procuramos obter as características dos artigos analisados e, em consequência, identificar a representatividade dessas pesquisas neste estudo.

T abela 2 - Características dos estudos indexados no MEDLINE no período de 2000 a 2010 utilizando os descritores enfermagem, obesidade e adolescência. Fortal eza, CE , 2010.

\begin{tabular}{lcc}
\hline Características dos estudos & $\mathbf{n}$ & \% \\
\hline Obesidade como causa de transtorno & & \\
mental & 2 & 10 \\
Co-morbidades entre adolescentes obesos & 5 & 25 \\
Prevalência da obesidade entre & & \\
adolescentes obesos & 5 & 25 \\
Causas da obesidade entre adolescentes & & \\
obesos & 3 & 15 \\
Intervenções da obesidade em adolescentes & 5 & 25 \\
\hline Total & $\mathbf{2 0}$ & $\mathbf{1 0 0}$ \\
\hline
\end{tabular}

E m relação às características dos estudos, $25 \%(5)$ das pesquisas se relacionavam às co-morbidades, à prevalência da obesidade e às intervenções em adolescentes obesos; $15 \%$ (3) dos estudos tratavam das causas; e 10\% (2) abordavam a obesidade como fator desencadeante de transtornos mentais. 0 risco de desenvolver doenças aumenta com o grau de sobrepeso. 0 ganho de peso surge como efeito direto do uso de medicações antipsicóticas $^{(32)}$.

F oi considerando relevante, na T abela 3, a discussão do problema de cada pesquisa com enfoque na temática que foi proposta a aproximação com os objetivos desta pesquisa.

Tabela 3 - Tema das pesquisas indexadas no M ED LINE no período de 2000 a 2010 utilizando os descritores enfermagem, obesidade e adolescência. Fortaleza, CE , 2010.

\begin{tabular}{|c|c|c|}
\hline T ema & $\mathrm{n}$ & $\%$ \\
\hline $\begin{array}{l}\text { O besidade juvenil } \\
\text { O besidade juvenil e recomendações } \\
\text { de enfermagem sobre a promoção da } \\
\text { saúde } \\
\text { A ções da obesidade juvenil com enfoque } \\
\text { na prática de enfermagem } \\
\text { Recomendações da prática de enfermagem } \\
\text { sobre a promoção da saúde }\end{array}$ & 11 & 55 \\
\hline T otal & 20 & 100 \\
\hline
\end{tabular}


E m relação ao enfoque da temática do estudo, a de maior frequência identificada foi obesidade juvenil com recomendações da prática de enfermagem sobre a promoção da saúde, com $55 \%$ (11) dos estudos, mostrando que a atuação do enfermeiro junto aos adolescentes obesos necessita de ações de promoção da saúde que cumpram a finalidade de conduzir a práticas efetivas, a fim de que as estratégias direcionadas a essa população alcancem os resultados esperados.

A identificação da área temática propicia 0 melhor manejo junto à população investigada e as possibilidades de atuação da enfermagem, requerendo maior participação desses profissionais com embasamento científico.

Consideramos importante anal isar a área temática das investigações desenvolvidas, que serão apresentadas na T abela 4. Salientamos que essas temáticas foram desenvolvidas pelos próprios pesquisadores, baseados no enfoque de cada estudo.

T abela 4 - Área temática dos estudos indexados no M EDLINE, no período de 2000 a 2010, utilizando os descritores enfermagem, obesidade e adolescência, segundo a área temática. Fortaleza, CE , 2010.

\begin{tabular}{lrr}
\hline Área temática & $\mathbf{n}$ & $\mathbf{\%}$ \\
\hline D epressão em adolescentes obesos & 1 & 5 \\
O besidade juvenil em escolares & 1 & 5 \\
Problemática da obesidade juvenil & 6 & 30 \\
Problema intelectual relacionado à obesidade & 4 & 20 \\
F amília & 1 & 5 \\
T er apêutica & 5 & 25 \\
A tuação do enfermeiro & 2 & 10 \\
\hline T otal & $\mathbf{2 0}$ & $\mathbf{1 0 0}$ \\
\hline
\end{tabular}

0 enfoque da área temática se deu, principalmente, na problemática da obesidade juvenil e sua terapêutica, seguido pelo problema intelectual relacionado com a obesidade. A área temática de menor prevalência foi a atuação de enfer magem, seguida por família, depressão em adolescentes obesos, e obesidade juvenil em escolares.

As evidências dos estudos realizados com adolescentes obesos têm mostrado o grande potencial de risco que a obesidade apresenta nesses sujeitos em desenvolver problemas psicológicos e de saúde. Observamos, a partir da análise dos estudos, a necessidade de uma atuação da enfermagem que considere os aspectos emocionais e psicológicos do sujeito e da família, favorecendo, desta forma, uma terapêutica norteada por ações de promoção da saúde que compreendam a ansiedade, a depressão e as dificuldades comportamentais e intelectuais como consequência da obesidade ${ }^{(33)}$.

A o analisar a estrutura teórica das publicações, observamos que $10 \%$ (2) dos estudos apresentaram quadro teórico, 5\% (1) citaram o referencial de Enfermagem Familiar na Promoção da Saúde, $5 \%$ (1) a T eoria da A prendizagem, e $90 \%$ (18) não utilizaram nenhum referencial teórico.

As teorias e os modelos conceituais per mitem aos pesquisadores organizar os achados da pesquisa e tor ná-los mais significativos e inter pretáveis, ou seja, servem para explicar os resultados da pesquisa(34).

Posteriormente, analisaremos os artigos que apresentavam revisão de literatura para embasar o problema proposto, destacando-se que $100 \%$ (20) apresentaram revisão de literatura sobre o problema a ser investigado. Revisar a literatura existente e incluí-la no estudo constitui um passo crítico do processo de pesquisa. 0 relato conciso do conteúdo revisado, como crítica aos pontos fortes e lacunas existentes na literatura, é um processo que possibilita apresentar o conhecimento atualizado sobre os problemas de pesquisa ${ }^{(31)}$.

$E m$ relação às considerações finais e conclusões dos artigos que fizeram parte da amostra desta revisão, 85\% dos estudos (17) apresentaram, e $15 \%$ (3) não apresentaram as conclusões respondendo aos objetivos propostos nos estudos, como é esperado no desenvolvimento de um trabalho científico.

A presentamos, abaixo, uma síntese dos principais resultados obtidos nos artigos: 15\% (3) mostraram a necessidade do desenvolvimento de mais pesquisas com intervenções eficazes para a preservação da saúde psicossocial de adolescentes obesos; $20 \%$ (4) apresentaram a necessidade de prevenir e tratar a obesidade infantil e adolescente, dada a atual epidemia de obesidade na população pediátrica e pelo fato de a prevenção apresentar resultados promissores; médicos e enfermeiros precisam trabalhar em parceria com funcionários e cuidadores para criar estilos de vida mais ativos foram resultados apresentados em $20 \%$ (4) dos estudos; e $25 \%$ (5) dos estudos evidenciaram a importância de pediatras e enfermeiros se tornarem conhecedores de estratégias e recomendações para os adolescentes obesos, comprometendo-se com 
a solução do problema da iminente epidemia de obesidade.

Por fim, observamos que os estudos apresentavam recomendações sobre a prática de enfermagem. D entre os estudos que apresentaram as recomendações, $75 \%$ (15) propõem a realização de pesquisas e sugestões específicas para a mudança na prática e melhoria das ações de enfermagem.

Tendo em vista as recomendações das pesquisas que tratam da obesidade juvenil, se faz necessária a construção de novos estudos que priorizem o tratamento da obesidade na população juvenil. Desta forma, é salutar a produção científica que auxilie os programas de orientação e implementação de estratégias de intervenção direcionadas às necessidades desse público.

Os estudos utilizados na produção desta revisão apresentaram coerência com os objetivos propostos, com o método e a referência utilizados. $E$ também fizeram sugestões para que mudanças nas práticas de enfermagem se tornem uma realidade para atingir a população-alvo. Enfocaram quais as implicações desses resultados, mas não mostraram a viabilização na prática clínica.

A presentamos, a seguir, as recomendações identificadas nos estudos selecionados para melhor visualização dos tipos de considerações para a prática de enfermagem apresentadas pelos pesquisadores: $10 \%$ (2) dos estudos referiram que os profissionais de enfermagem e outros prestadores de cuidados de saúde devem ser mais diligentes na seleção e documentação da obesidade na população pediátrica; $5 \%$ (1) informaram que as enfermeiras da escola têm o potencial para reduzir os atos agressivos de adol escentes vitimados pel o excesso de peso através da implementação de intervenções apropriadas; 35\% (7) dos estudos abordaram a importância de 0 enfermeiro reconhecer os adolescentes que estão acima do peso, pois eles possuem um problema de saúde com riscos de complicações a longo prazo; prevenção e tratamento eficazes requerem uma abordagem integrada; $15 \%$ (3) dos estudos afirmaram que 0 enfermeiro pediatra precisa incentivar os pais e grupos de interesse para a inclusão de cantinas escolares com opções de oferta de alimentos saudáveis, excluindo as oferecidas por for necedores de fast-food; e, ainda, 10\% (2) enfatizaram a importância da educação continuada pelos enfermeiros acerca da prevenção e tratamento da obesidade juvenil.
O caminho percorrido no desenvolvimento do estudo faz com que resultados possam ser utilizados como evidência científica com implicações para a prática profissional. Alguns autores, a partir dos resultados, fazem recomendações específicas para a mudança da prática clínica com a identificação da população estudada; a representatividade das pesquisadas selecionadas, o tempo de coleta de dados, a justificativa da escolha do método para o alcance dos objetivos, e até mesmo 0 preparo dos pesquisadores para o desenvolvimento do estudo são fatores que determinam o rigor de um estudo científico.

Percebemos que a enfermagem apresenta grande interesse em pesquisa quantitativa, 0 que leva a identificar as características da população estudada, facilitando um direcionamento das ações de enfermagem acerca da obesidade juvenil.

A revisão integrativa aparece como alternativa para sintetizar o conhecimento obtido por pesquisa, e essas revisões têm priorizado as evidências obtidas por ensaios clínicos randomizados, para determinar as intervenções de saúde ${ }^{(31)}$.

$\mathrm{N}$ a síntese do conhecimento não são necessários apenas dados quantitativos, mas também uma compreensão crítica de evidências geradas por outros estudos relevantes para a prática clínica ${ }^{(32)}$. Foi o modelo de hierarquia das evidências que colocou os dados quantitativos em oposição aos dados obtidos em estudos qualitativos, que também produzem importante influência nas intervenções de saúde.

A abordagem da pesquisa qualitativa tornase fundamental para responder as questões relacionadas aos sentimentos dos indivíduos em relação à experiência de saúde e doença e, principalmente, na enfermagem com enfoque em obesidade juvenil para entender as melhores intervenções implementadas com vistas à promoção da saúde.

Os resultados da pesquisa evidenciaram os seguintes tópicos de interesse: educar enfermeiros que atuem na escola sobre a identificação, intervindo na vitimização de adolescentes obesos; buscar desenvolver as intervenções baseadas em evidências para o tratamento e prevenção da obesidade juvenil na prática; envolver ativamente a sociedade na escolha de atividade física e participar de uma intervenção baseada nos pais com seus filhos, em um esforço para aumentar a consciência da importância do exercício físico na adoles- 
cência; avaliar o impacto da promoção da saúde sobre a perda de peso dos participantes; descrever a avaliação de problemas de saúde relacionados à obesidade, bem como orientações científicas e estratégias de intervenção baseadas em pesquisas para diminuir a obesidade na adolescência.

A identificação dos objetivos favoreceu 0 aumento do conhecimento para a prática da enfermagem com enfoque na obesidade juvenil, definindo áreas prioritárias, facilitando o estudo para futuras pesquisas, melhorando o preparo dos enfermeiros para objetivar ações de promoção da saúde junto a essa problemática.

\section{CONSIDERAÇÕES FINAIS}

A pesar do número pequeno de artigos encontrados sobre a temática proposta, percebemos que a maioria dos estudos acerca desse tema foi publicada em um periódico próprio para a população de crianças e adolescentes, o Pediatric N ursing J ournal, com 35\% (7) das pesquisas analisadas, 0 qual concentra pesquisas e estratégias desenvolvidas no intuito de solucionar a problemática que envolve a obesidade juvenil.

A realização desta pesquisa evidencia a necessidade da construção do conhecimento científico da enfermagem com enfoque na promoção da saúde na obesidade juvenil, contribuindo para que as pesquisas da enfermagem que visam à promoção da saúde em adolescentes obesos cumpram a sua finalidade de conduzir a práticas e intervenções efetivas e de qualidade, a fim de que as ações de enfermagem direcionadas a essa população sejam implementadas e alcancem resultados efetivos.

Consideramos que os objetivos propostos pelo estudo tenham sido alcançados e que a visualização do risco cumulativo que a obesidade juvenil apresenta em tornar o sujeito um adulto obeso é um dado precioso para o planejamento das ações de saúde no tratamento individualizado dessa população, implementando estratégias de cuidados, capacitando a equipe multidisciplinar no intuito de obter uma melhor adequação das ações frente a esse problema de saúde.

D entre as limitações identificadas nos estudos analisados, notamos que, apesar das contribuições dadas pelas pesquisas na área da enfermagem sobre o tema em questão, ainda não existem soluções reais para o controle desse problema. Sugeri- mos, então, que além do aprofundamento da temática, possamos também criar estratégias de promoção da saúde no combate à obesidade juvenil.

\section{REFERÊ NCIAS}

1 Valdelamar L, Rodríguez M , Bermúdez V, Leal E, Bermúdez F, Cabrera M. T ratamiento farmacológico de la obesidad: presente, pasado y futuro. AVFT. 2007; 26(1):10-20

2 Kuschnir M CC, M endonça GAS. Risk factors associated with arterial hypertension in adolescents. J Pediatr. 2007;83(4):335-42.

3 M ello ED, Luft VC, M eyer F. O besidade infantil: como podemos ser eficazes? J Pediatr. 2004;80(3):173-82.

4 Faria ER, Fransceschini SCC, Peluzio M CG, Priore SE. M etabolic syndrome in adolescents: an update. N utrire Rev Soc Bras Aliment N utr. 2009;34(2):179-94.

5 Sabrina R N . Victimization of obese adolescents. J Sch N urs. 2006;22(4):202-6.

6 Jerum A, M elnyk BM . E ffectiveness of interventions to prevent obesity and obesity: related complications in children and adolescents. Pediatr N urs. 2001;27(6): 606-10.

7 Simmons A, M avoa H M , Bell AC, Courten M , Schaaf $D$, Schultz J, et al. Creating community action plans for obesity prevention using the ANGELO (A nalysis $G$ rid for Elements Linked to O besity) Framework. H ealth Promot Int. 2009;24(4):311-24.

8 M endes KDS, Silveira RCCP, G alvão CM . Revisão integ rativa: método de pesquisa para a incorporação de evidências na saúde e na enfermagem. T exto Contexto Enferm. 2008;17(4):758-64.

9 Garbin LM , Rodrigues CC, Rossi LA, Carvalho EC. Classificação de resultados de enfermagem (NOC): identificação da produção científica relacionada. Rev G aúcha E nferm. 2009;30(3):508-15.

10 Silveira CS. A pesquisa em enfermagem oncológica no Brasil: uma revisão integrativa [ dissertação] . Ribeirão Preto: E scola de E nfermagem, U niversidade de São Paulo; 2005.

11 Introduction to quantitative research in nursing. In: Burns N, G rove SK. T he practice of nursing research: conduct, critique and utilization. Philadel phia: W. B. Sanders; 2001, p. 19-36. 
12 Jevitt CM. Weight management in gynecologic care.J M idwifery W omens H ealth. 2005;50(5):427-30.

13 Calderon KS, Y ucha CB, Schaffer SD. Obesity related cardiovascular risk factors: intervention recommendations to decrease adolescent obesity. J Pediatr N urs. 2005;20(1):3-14.

14 Christine A B, M ona A E, Sandra LU, Janet CM , Lynne E H, G wen J, et al. Student nurses participate in public health research and practice through a school: based screening program. Public H ealth N urs. 2005; 22(3):260-6.

15 D avid M, Roy M C, Gordon M. Obesity in people with intellectual disabilities: the impact of nurseled heard screenings and health promotion activities. A m J H ealth Promot. 2003;41(2):147-53.

$16 \mathrm{E}$ dward EY. An activity calendar program for children who are overweight. Pediatr N urs. 2003;29 (1):19-22.

$17 \mathrm{E}$ ric $\mathrm{AH}$. A primer on early childhhod obesity and parental influence. Pediatr N urs. 2003;29(1):13-16.

18 Camden SG. A captive condition: childhood obesity. N urs M anage. 2009;40(2):25-31.

19 Berry D, G alasso P, M elkus G, G rey M. Obesity in youth: implications for the advanced practice nurse in primary care. J A m A cad N urse Pract. 2004;16(8): 326-34.

20 Covington CY, Cybulski M J, D avis T L, Duca GE, Farrell EB, Kasgorgis M L. Kids on the move: preventing obesity among urban children. Am J Nurs. 2001;101(3):73-82.

21 Roberts SO. T he role of physical activity in the prevention and treatment of childhood obesity. Pediatr N urs. 2000;26(1):33-6, 39-41.

22 Buiten C, M etzger B. Childhood obesity and risk of cardiovascular disease: a review of the science. Pediatr N urs. 2000;26(1):13-8.

$23 \mathrm{~N}$ ancy R. Considerations for patients who have eating disorders. AORN J. 2010;31(1):11-7.

24 Small L, Anderson D, Sidora-Arcoleo K, Cleveland BG. Pediatric nurse practitioners' assessment and management of childhood overweight obesity: results from 1999 and 2005 cohort surveys. J Pediatr Health Care. 2009;23(4):231-41.

25 Cleveland BG, A rcoleo KS, Keesing H, G ottesman $M$ M , Brady M. Changes in nurse practitioners' knowledge and behaviors following brief training on the healthy eating and activity together (HEAT) guidelines. J Pediatr Health Care. 2009;23(4):22230.

26 Landis AM, Parker KP, D unbar SB. Sleep, hunger, satiety, food cravings, and caloric intake in adolescents. J N urs Scholarsh. 2009;41(2):115-23.

27 T ilghman J. Obesity and diabetes in African American women. ABN F J. 2003;14(3):66-8.

28 Ross HM , Laws R, Reckless LM . Evaluation of the Counterweight Programme for obesity management in primary care: a starting point for continuous improvement. Br J G en Pract. 2008;58(553):548-54.

29 Berry D, M elkus G, Savoye M, Grey M. An intervention for multiethnic obese parents and overweight children. A ppl N urs Res. 2007;20(2):63-71.

300 hlsen RI, Peacock G, Smith S. D evel oping a ser vice to monitor and improve physical health in people with serious mental illness. J P sychiatr $\mathrm{M}$ ent $\mathrm{H}$ ealth N urs. 2005;12:614-9.

31 Streubert HJ. Avaliação do relatório de pesquisa qualitativa. I n: L obiondo G , H aber J. Pesquisa em enfermagem: métodos, avaliação crítica e utilização. Rio de Janeiro: G uanabara Koogan; 2001. p. 255-67.

32 Grey M. Desenhos experimentais e quase experimentais. In: L obiondo G, H aber J. Pesquisa em enfermagem: métodos, avaliação, crítica e utilização. 4a ed. Rio de Janeiro: G uanabara Koogan; 2001. p. 98109.

33 L uiz AM AG, Gorayeb R, Liberatore Junior RDR, D omingos NAM . D epressão, ansiedade e competência social em crianças obesas. E stud P sicol. 2005;10 (1):35-9.

34 Introduction to quantitative research in nursing. In: Burns N, G rove SK. T he practice of nursing research: conduct, critique and utilization. P hiladel phia: W. B. Sanders; 2001. p. 19-36.

Recebido em: 10/ 09/2010

A provado em: 18/ 03/ 2011

\section{Endereço do autor / Dirección del autor / Author's address:}

I zaildo T avares Luna

Rua Carlos Vasconcelos, 1847, ap. 303, A Ideota

60115-171, Fortaleza, CE

E-mail: izaildo@ufc.br 